\title{
A simple model for a radiatively driven metallic wind in A0 stars
}

\author{
J. BABEL
}

Service d'Astrophysique, Centre d'Etudes de Saclay, 91191 Gif-sur-Yvette, France

Abstract. Litle is known about the wind of main sequence A7 to B4 stars. We here investigate the case of a radiatively driven wind for an A0 star and obtain stringent limits on the mass loss rate. We also show that frictional heating lead to the presence of a chromospheric region very near the photosphere.

\section{A radiatively driven metallic wind in A stars?}

For main sequence stars with $T_{\text {eff }} \lesssim 14000 \mathrm{~K}$, the maximum radiative acceleration in expanding atmospheres, $g_{r a d, t o t}^{\max }$, is always smaller than gravity (Abbott 1982) and there is no possibility of homogeneous radiatively driven winds. Individually however, metals receive a large radiative acceleration which can overcome gravity even in the photosphere (Babel \& Michaud 1991). Approximating the radiative acceleration transmitted to metals (as a whole), $g_{r a d, Z}^{\max }$, by $g_{\text {rad,tot }}^{\max } / Z$, with $\mathrm{Z}$ the mass fractions of metals, we obtain that $g_{r a d, Z}^{\max }>g$ and that a metallic wind can potentially be present, but cannot drag $\mathrm{H}$ and $\mathrm{He}$ in the subsonic domain.

The individual momentum equation for a stationary multicomponent flow is:

$$
\frac{\partial_{r} V_{i}}{V_{i}}\left(a_{i}^{2}-V_{i}^{2}\right)+2 \frac{a_{i}^{2}}{r}-\frac{g}{r^{2}}+g_{r a d, i}+\frac{z_{i} E}{m_{i}}-\sum_{j \neq i} n_{j} \frac{R}{k T} \frac{z_{i} z_{j}}{m_{i}} g\left(X_{i j}\right) \frac{V_{i}-V_{j}}{\left|V_{i}-V_{j}\right|} \equiv 0(1)
$$

and the global energy equation is (Braginskii 1965), for $T_{i}=T \forall i$,

$$
\begin{aligned}
& \sum_{i \neq e} n_{i} V_{i} k T\left(\frac{3}{2} \frac{\partial \ln (T)}{\partial r}\left(1+z_{i}\right)+\frac{5}{2} \frac{\partial z_{i}}{\partial r}-z_{i} \frac{\partial \ln \left(n_{e}\right)}{\partial r}-\frac{1}{n_{i}} \frac{\partial n_{i}}{\partial r}\right)= \\
& -\sum_{i} \operatorname{div} q_{i}+Q_{r a d}+Q_{\text {fric }}
\end{aligned}
$$

We make here the approximation of Maxwellian distribution for all components, nebular approximation for the ionisation equilibrium and assume that $g_{r a d, i}$ is not a function of $\partial_{r} V_{i}$. This last approximation is justified by the very small optical depth of the medium. In the case of $\mathrm{A} 0$ stars, $\mathrm{H}$ and $\mathrm{He}$ can be considered as being in hydrostatic equilibrium. In this case and if we consider all metals as one average population (label $\mathrm{Z}$ ) there is only one critical point for $V_{Z}^{2}=k T / m_{Z}$. It does not fix the mass loss rate. The mass loss rate is fixed instead by the populations of each species at the lower boundary (photosphere). 

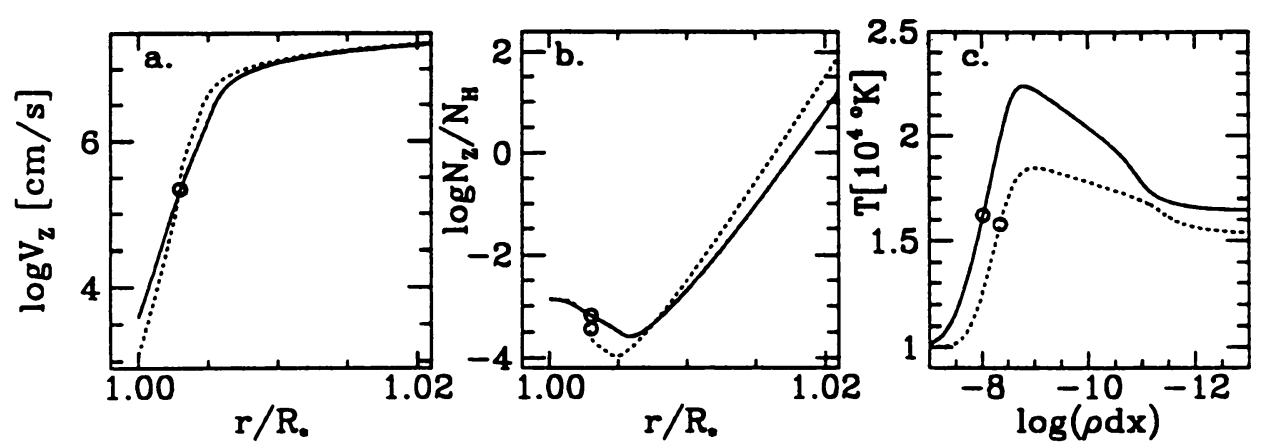

Fig. 1. a. Velocity of metals vs radius. Solid line (model $A$ ), dotted line (model $B$ ). b. Same as in (a) but for the concentration of metals. c. Temperature as a function of the column density. A circle indicates the position of the critical point in the two models.

\section{Results and discussion}

Equations 1 and 2 were solved simultaneously for a ZAMS star with $T_{\text {eff }}=$ $10000 \mathrm{~K}, \log g=4.37$, for a force multiplier $M_{\max }=10^{2.7}$ (Abbott 1982) and for lower boundary conditions: $T^{\circ}=T_{\text {eff }}, N_{H}^{\circ}=10^{9} \mathrm{~cm}^{-3}, Y^{\circ}=Y_{\odot}$ and $Z^{\circ}=Z_{\odot}$ (we consider the mean metallic atom as an iron atom). Model $A$ is for a case of constant radiative acceleration $g_{r a d, Z}=g_{r a d, Z}^{\max }\left(R_{*} / r\right)^{2}=$ $1.710^{5}\left(R_{*} / r\right)^{2}$ and model $B$ is for a case case where $g_{r a d, Z}$ varies with $V_{m}$ in a way which simulates the effect of Doppler shift on the radiative acceleration.

Results are shown in Fig. 1. The metallic mass loss rate corresponding to the 2 models are: $(A) 6.510^{-16} \mathrm{M}_{\odot} \mathrm{yr}^{-1},(B) 1.910^{-16} \mathrm{M}_{\odot} \mathrm{yr}^{-1}$. Any additional decrease, relative to the cases considered here, of the radiative acceleration or any additional coupling of the gases below (or at) the critical point decreases the mass loss rate. The estimates from our simple model give thus an approximate upper limit on the mass loss rate due to radiative pressure. They are of similar order as obtained from the diffusion theory (Babel 1992).

Even if $M$ is very small, the frictional heating, $Q_{\text {fric }}$, plays a major role, as in $\tau$ Sco (Springmann \& Pauldrach 1992), and leads to a large increase of the gas temperature near the critical point. It could thus lead, for A and B stars, to much larger EUV radiation than expected from hydrostatic (LTE or NLTE) models in radiative equilibrium. This could help to solve the problem caused by the EUV flux of $\beta$ Cma (Cassinelli et al., this volume) .

\section{References}

Abbott, D. C.:1982, ApJ 259, 282

Babel, J.:1992, A\&A 258, 449

Babel, J., Michaud, G.:1991, ApJ 366, 560

Braginskii, S. I.:1965, in Reviews of Plasma Physics, New York, p. 205

Springmann, U. W. E, Pauldrach, A. W. A.: 1992, A\&A 262, 515 